\title{
Is early mobilization associated with functional improvement in acute middle cerebral artery ischemic stroke treated with recombinant tissue plasminogen activator? A proof of concept and retrospective single-center study
}

\author{
Hsiao-Ching Yen ${ }^{1}{ }^{*}$, Jiann-Shing Jeng ${ }^{2}$, Wen-Ying Chuang ${ }^{1}$, Wen-Shiang Chen ${ }^{3}$
}

\author{
${ }^{1}$ Division of Physical Therapy, \\ Department of Physical Medicine and \\ Rehabilitation, National Taiwan \\ University Hospital, Taipei, Taiwan \\ ${ }^{2}$ Stroke Center \& Department of \\ Neurology, National Taiwan University \\ Hospital, Taipei, Taiwan \\ ${ }^{3}$ Department of Physical Medicine and \\ Rehabilitation, National Taiwan \\ University Hospital, Taipei, Taiwan \\ *Correspondence \\ jassicayen@yahoo.com.tw \\ (Hsiao-Ching Yen)
}

\begin{abstract}
Background: The benefit of commencing early mobilization for patients after ischemic stroke (IS) is well established. However, the timing and benefits of early mobilization in recombinant tissue plasminogen activator (rtPA)-treated patients remain mostly undefined.

Methods: In this retrospective observational cohort study, we analyzed rtPA-treated patients after a first-episode middle cerebral artery (MCA)-IS, that were admitted in a Stroke Center, using electronic medical records during the period of January 1, 2015 through December 31,2017. These patients who received a standard early rehabilitation during the stay in the Stroke Center served as the control group. We examined the association between early mobilization via early rehabilitation within 24-72 hours and activities of daily living and postural control improvements, as indicated by the Barthel Index (BI) effectiveness and the Postural Assessment Scale for Stroke (PASS) effectiveness, respectively.

Results: Total 60 patients with a first MCA-IS treated after rtPA were analyzed. Multiple linear regression analysis indicated that BI and PASS effectiveness were positively correlated with not only age and the stroke severity at 24 hours after rtPA treatment but commencing early mobilization within $24-72$ hours after stroke in patients with a first MCA-IS treated after rtPA.

Conclusions: This study concludes that early mobilization may be associated with an acceleration of functional improvement in patients with a first MCA-IS treated with rtPA within four weeks after the onset.
\end{abstract}

\section{Keywords}

Acute stroke; Early mobilization; Thrombolytic therapy; Functional outcome; rtPA

\section{Introduction}

Even though the treatments for stroke have evolved rapidly in recent years, the stroke remains a major global cause of mortality and disability. The middle cerebral artery (MCA) was found to be involved in more than two-thirds of all cases of brain infarcts included in a number of stroke registries. Systemic treatment with intravenous thrombolysis (IVT) of recombinant tissue plasminogen activator ( $\mathrm{rPA})$ is the key factor for the prognosis of acute MCA ischemia [1] and achieve recanalization pharmacologically [2]. However, rtPA bears the potential for adverse effects, such as cerebral hemorrhage, and its protocols even include a recommendation for 24-48 hours of bed rest after treatment [3]. The optimal timing of early mobilization (EM) in rtPA-treated patients remains largely undefined.

EM after ischemic stroke (IS) might prevent immobilityrelated complications [4-6], promote brain recovery [7] and contributed to an improved functional outcome $[8,9]$. Preclinical studies also provided evidence that intervention within the first 72 hours of stroke event decreases inflammatory cytokines [10, 11], tightens the blood-brain barrier [12], suppresses apoptosis [13, 14], increases brainderived neurotrophic factor (BDNF) levels [15], and promote neurogenesis $[14,16]$. While some clinicians may have concerns that EM (i.e., involving upright activity) might inhibit the reperfusion of salvageable penumbral tissue [17], no significant differences were found in cerebral blood flow velocity between patients assessed at 6 to 16 hours after stroke and controls tested in $70^{\circ}, 45^{\circ}, 0^{\circ}$, and $-15^{\circ}$ positions [18]. Thus, commencement of EM soon after an IS may not be harm.

However, there is limited information on the specific timing of starting early mobilization after intravenous rtPA treatment that would potentially optimize the recovery during the acute period [19-21]. Although, one large retrospective study of 
subjects treated with intravenous rtPA in Japan showed an association between starting early rehabilitation within three days of admission and functional independence at hospital discharge [19], the data were taken from the diagnosis procedure combination database [22]. Therefore, that study did not provide detailed information about the used rehabilitation programs, the exact timing of admission, rtPA dose, the precise lesion locations, or stroke severity upon admission [19]. In addition, the inclusion criteria in AVERT (A Very Early Rehabilitation Trial) were included IS patients treated after rtPA [21], but the protocol was characterized by high-frequency early mobilization within the first 24 hours and by the lack of the National Institutes of Health Stroke Scale (NIHSS) score at 24 hours post-rtPA [20, 21]. Besides, very EM protocol within 24 hours of stroke onset may also be associated with a worse outcome at the three-months post-stroke time point $[7,8]$. Therefore, it is needed to establish the impact of the EM within 24-72 hours after stroke onset that treated with rtPA on the functional outcomes, compared to the standard early rehabilitation in which the mobilization starts after ICU discharge.

Therefore, the aim of the study was to assess whether beginning the early mobilization within 24-72 hours after stroke in MCA-IS patients was associated with improved functional gain after IVT with rtPA treatment by using daily medical records and daily assessment reports of rehabilitation built into the electronic medical records (EMR) of National Taiwan University Hospital (NTUH).

\section{Methods}

\subsection{Study population}

This retrospective cohort study covering the period from January 1, 2015, through December 31, 2017 was conducted in the 12-bed stroke center of NTUH. The criteria for admission to the stroke center included being an IS patient receiving thrombolytic therapy, which was approved at a standard dose of 0.9 $\mathrm{mg} / \mathrm{kg}$ or a modified dose of $0.6 \mathrm{mg} / \mathrm{kg}$ within three hours of stroke onset. The study was approved by the Institutional Review Board (IRB) of NTUH (ID number: 201801125RINA).

The inclusion criteria for participants in the study were: patient experienced a first MCA-ischemic stroke that was diagnosed using conventional MRI or perfusion CT scan; patient was treated IVT with rtPA within 3 hours of onset without intra-arterial rescue therapy; patient was fully activities of daily living (ADL) independent before the stroke; a NIHSS score of $<25$ at admission; rehabilitation programs started within 3 days of the stroke; and patient had related complete prognosis records within 30 days of onset. The exclusion criteria were as follows: patients admitted for recurrent ischemic stroke, brain tumor, and other previous central nervous system dysfunction (for example, Parkinson's disease, dementia, etc.); and patient found to have an infarction located outside the area of the MCA during the same admission.

\subsection{Exposure of interest}

According to the content of rehabilitation programs recorded in EMR during the stroke center stay, the participants were divided into (1) the standard early rehabilitation (SER) group and (2) the early mobilization group.

For the SER group, a standard program started within 3 days of the stroke, but out-of-bed activities were started after ICU discharge. SER programs included positioning, range of motion exercises, in-bed mobilization, facilitation exercises, and caregiver education. However, since the effectiveness of EM for stroke have been confirmed [23], the rehabilitation team in stroke center started to commence EM within 24-72 hours after ischemic stroke since June 2016 (based on stable vital signs including $\mathrm{SBP}<180 \mathrm{mmHg}$ in resting; resting $\mathrm{HR}<130 \mathrm{bpm}$; no hydrocephalus before intervention and no early deterioration within 24 hours after stroke). Therefore, we identified the stroke patients who received treatment involving task-specific activities, such as sitting unsupported out of bed, and standing, within 24-72 hours of the stroke onset, and recorded the programs in EMR as the EM group $[8,9]$. Both groups received routine rehabilitation treatment performed by trained physical therapists based on the hospital policy, generally lasting about 30 minutes a day, for five days or until discharged.

\subsection{Data source and covariates}

In the NTUH Stroke Center, the patients received intensive medical, nursing, and early rehabilitative care. Before early rehabilitation, the patients received full functional and postural evaluations [24, 25], which were usually performed by a physical therapist. The rehabilitation program and the prognosis were recorded every day in the EMR. Besides, the BI total scores were recorded after every fifth intervention session in progress reports. Subsequently, if a patient was not transferred to the rehabilitation ward and was discharged from the neurological ward, the patient was typically scheduled to come back to the clinic for an evaluation at one month after the stroke onset.

In the study, age, sex, body height and weight, diagnosis, treatment methods, and the NIHSS score at admission and at 24 hours post-rtPA for each enrolled patient were collected. Using the parameters quantified were length of stay in stroke center, history of vascular risk factors, in-hospital post-thrombolysis complications, malignant stroke, and uncontrolled atrial fibrillation $[26,27]$. The comorbidities were identified using the codes for each patient [22]. The Charlson comorbidity index (CCI) was used to evaluate the accumulation of comorbidities $[19,28]$. The total scores of the BI and PASS at the initial intervention after admission was recorded and calculated.

\subsection{Outcome measures}

The variables recorded included BI [25] and PASS [24] total scores at four weeks after the stroke. A total BI score to measure basic ADL ranges from 0 to 100 , including feeding, bathing, grooming, dressing, bowel movements, bladder control, toilet use, transfers, mobility, and stair usage [25]. The PASS contains 12 four-level items of varying difficulty with a maximum score of 36 and is used to assess a patient's ability to maintain or change a given lying, sitting, or standing posture. The primary outcome measure of this study was the BI effectiveness, expressed as a percentage, reflecting the 
TA B L E 1. Characteristics of patients with middle cerebral artery ischemic stroke after rtPA treatment

Characteristic

Sex (Male)

Age upon admission, mean \pm SD

Lesion site (Left)

Body mass index $\left(\mathrm{kg} / \mathrm{m}^{2}\right)$, mean $\pm \mathrm{SD}$

rtPA dose (Standard), n (\%)

Charlson Comorbidity Index, n (\%)

0

1

2

3

$\geq 4$

The length of hospital stay (day), mean \pm SD

The length of ICU stay (day), mean \pm SD

NIHSS score upon admission, mean \pm SD

NIHSS score at 24 hours after rtPA treatment, mean \pm SD

Complications during hospitalization, $\mathrm{n}(\%)$

Symptomatic intracerebral hemorrhage

Myocardial infarction

Early seizure

Infection

Uncontrolled atrial fibrillation

BI upon admission (score), mean $\pm \mathrm{SD}$

PASS upon admission (score), mean \pm SD

$\mathrm{BI}$ effectiveness, mean $\pm \mathrm{SD}$

PASS effectiveness, mean \pm SD

Initial out-of-bed mobilization after stroke (day), mean \pm SD
Standard early rehabilitation $(\mathrm{N}=30)$ Early mobilization $(\mathrm{N}=30)$

$\begin{array}{cc}12(40 \%) & 16(53.3 \%) \\ 70.5 \pm 11.7 & 63.7 \pm 15.3 \\ 15(50.0 \%) & 19(63.3 \%) \\ 24.7 \pm 4.4 & 25.1 \pm 6.7 \\ 19(63.3 \%) & 10(33.3 \%)\end{array}$

$10(33.3 \%)$

$19(63.3 \%)$

$11(36.67 \%)$

$7(23.3 \%)$

$6(20.0 \%)$

$3(10.0 \%)$

$2(6.7 \%)$

$0(0 \%)$

$1(3.3 \%)$

$1(3.3 \%)$

$35.7 \pm 18.3$

$17.5 \pm 12.9$

$7 \pm 2.2$

$4.1 \pm 2.2$

$14.3 \pm 6.5$

$11.6 \pm 5.4$

$13.9 \pm 7.7$

$6.6 \pm 3.8$

$4(13.3 \%)$

$0(0 \%)$

$0(0 \%)$

$0(0 \%)$

$0(0 \%)$

$0(0 \%)$

$14(46.7 \%)$

$1(3.3 \%)$

0 (0)

$0(0)$

$2.8 \pm 6.3$

$9.1 \pm 5.4$

$2.9 \pm 1.7$

$7.2 \pm 6.3$

$0.5 \pm 0.4$

$0.9 \pm 0.2$

$0.6 \pm 0.3$

$0.9 \pm 0.1$

$9.4 \pm 3.9$

$2.7 \pm 0.6$

SD: standard deviation

NIHSS: National Institute of Health Stroke Scale

rtPA: recombinant tissue plasminogen activator

BI: Barthel Index

PASS: Postural Assessment Scale for Stroke

proportion of potential improvement vs. actually achieved improvement and was calculated using the following equation: ([BI at four weeks after the stroke onset - admission BI] / [maximum BI - admission BI]) [29]. Similarly, the second outcome measure of this study, the PASS effectiveness ([PASS at four weeks after the stroke onset - admission PASS] / [maximum PASS - admission PASS]).

\subsection{Statistical analysis}

All data were analyzed using the IBM SPSS software for Windows (version 17.0; SPSS Inc., Chicago, IL, USA). Continuous variables were summarized as the means \pm standard deviations if they were normally distributed. The chi-square or Fisher's exact tests for categorical variables was used and unpaired $t$-tests was used for continuous variables. $P$-values less than 0.05 were considered significant.
Several univariate regression analyses determined the best predictors for better BI or PASS effectiveness after four weeks of stroke onset, as well as to investigate the additional effects of time of mobilization commencement on the outcomes. Potential variables mentioned in previous studies, listed in Table 1 . All univariate potential predictors with a $P$-value $<0.1$ were entered into the multivariate model. Collinearity among potential variables was evaluated; variables with moderate to high intercorrelations $(r \geq 0.5$ or $r \leq-0.5)$ were regarded as collinear, and only one was entered into the regression model. Next, multiple linear regression analyses were used to assess the effects of early mobilization on BI or PASS effectiveness adjusted for selected variables. In the multiple linear regression models, a stepwise strategy was used to select the variables remaining in the final model. 
T A B L E 2. Univariate regression analysis results for Barthel Index effectiveness

\begin{tabular}{|c|c|c|c|}
\hline & Coefficient $(\beta)$ & $95 \% \mathrm{CI}$ & $P$-value $\left(<0.1^{*}\right)$ \\
\hline \multicolumn{4}{|l|}{ Rehabilitation type } \\
\hline Early standard rehabilitation & Reference & & \\
\hline Early mobilization & 0.39 & $(0.25,0.54)$ & $<0.001 *$ \\
\hline Age & -0.01 & $(-0.02,0.00)$ & $0.001 *$ \\
\hline \multicolumn{4}{|l|}{ Sex } \\
\hline Male & Reference & & \\
\hline Female & -0.12 & $(-0.30,0.06)$ & 0.18 \\
\hline \multicolumn{4}{|l|}{ Lesion site } \\
\hline Left & Reference & & \\
\hline Right & -0.10 & $(-0.28,0.07)$ & 0.26 \\
\hline Body mass index & 0.01 & $(-0.01,0.02)$ & 0.45 \\
\hline Duration of ICU stay & -0.07 & $(-0.10,-0.04)$ & $<0.001 *$ \\
\hline NIHSS score upon admission & -0.01 & $(-0.03,0.00)$ & 0.11 \\
\hline NIHSS score at 24 hours after rtPA treatment & -0.03 & $(-0.04,-0.02)$ & $<0.001 *$ \\
\hline \multicolumn{4}{|l|}{ rtPA dose } \\
\hline Standard dose & Reference & & \\
\hline Modified dose & 0.26 & $(0.09,0.42)$ & $0.003 *$ \\
\hline $\begin{array}{l}\text { Charlson Comorbidity Index } \\
\text { (as a continuous variable) }\end{array}$ & -0.09 & $(-0.18,-0.01)$ & $0.038^{*}$ \\
\hline
\end{tabular}

NIHSS: National Institute of Health Stroke Scale; rtPA: recombinant tissue plasminogen activator

\section{Results}

\subsection{Cohort characteristics}

During the study period, 171 of a total of 557 new admissions collated during a manual review of the EMRs were first-episode MCA ischemic patients. Ultimately, 72 met the inclusion criteria and were included in this study. The reasons for 12 further exclusions in the final analysis were death $(\mathrm{n}=$ 1 ) and incomplete data sets $(n=11)$. As a result, the analyzed 60 first-episode MCA-IS patients treated with rtPA, of whom 30 were included in the early mobilization group.

\subsection{Outcome data}

Table 1 shows the clinical characteristics of the MCA-IS with rtPA treatment. The average interval from stroke onset to the commencement of out-of-bed mobilization in the EM group (30 patients) was $2.70 \pm 0.6$ days, while that for the SER group (30 patients) was $9.43 \pm 3.9$ days $(P<0.001)$. Compared to the SER group, the mean BI and PASS scores as well as BI and PASS effectiveness, upon admission, were significantly higher, the mean NIHSS score at 24 hours after rtPA treatment was significantly lower, and the percentage using the standard rtPA dose was significantly lower in the EM group. There was no significant difference in age or mean NIHSS score upon admission between the two groups. Otherwise, infections were significantly less frequent in the EM group.
The results of univariate analyses are shown in Table 2 and Table 3, respectively. Two sociodemographic variables (age and $\mathrm{CCI}$ ) and several clinical stroke-related variables were significantly associated with the BI effectiveness and with PASS effectiveness. The potential predictors listed in Table 2 and Table 3 were put into the multiple regression analysis. The multiple linear regression analysis identified the role of early mobilization therapy as a significant positive factor affecting BI effectiveness (coefficient, 0.23; 95\% confidence interval (CI), 0.08 0.39; $P=0.004$; Table 4 ). In addition, age and the NIHSS score at 24 hours after rtPA treatment were also significant positive factors for BI effectiveness (Table 4). Together, these three variables accounted for $49 \%$ of the total variance.

Similarly, early mobilization therapy as a significant positive factor affecting PASS effectiveness (coefficient, 0.17; 95\% confidence interval (CI), 0.03 0.32; $P=0.019$, Table 4 ). In addition, age and the NIHSS score at 24 hours after rtPA treatment were also significant positive factors affecting PASS effectiveness. Together, these three variables accounted for $39 \%$ of the total variance.

\section{Discussion}

In this proof-of-concept study, the results demonstrated that beginning timely mobilization via early rehabilitation for patients with a first MCA-IS treated with rtPA in a stroke center was associated with better functional progress. A prompt 
TA B L E 3. Univariate regression analysis results for Postural Assessment Scale for Stroke effectiveness

\begin{tabular}{|c|c|c|c|}
\hline & Coefficient $(\beta)$ & $95 \% \mathrm{CI}$ & $P$-value $\left(<0.1^{*}\right)$ \\
\hline \multicolumn{4}{|l|}{ Rehabilitation type } \\
\hline Early standard rehabilitation & Reference & & \\
\hline Early mobilization & 0.29 & $(0.16,0.42)$ & $<0.001 *$ \\
\hline Age & -0.01 & $(-0.01,0.00)$ & $0.001^{*}$ \\
\hline \multicolumn{4}{|l|}{ Sex } \\
\hline Male & Reference & & \\
\hline Female & -0.11 & $(-0.26,0.04)$ & 0.14 \\
\hline \multicolumn{4}{|l|}{ Lesion site } \\
\hline Left & Reference & & \\
\hline Right & -0.09 & $(-0.24,0.06)$ & 0.26 \\
\hline Body mass index & 0.01 & $(-0.01,0.02)$ & 0.44 \\
\hline Duration of ICU stay & -0.05 & $(-0.08,-0.03)$ & $<0.001 *$ \\
\hline NIHSS score upon admission & -0.01 & $(-0.02,0.00)$ & $0.076^{*}$ \\
\hline NIHSS score at 24 hours after rtPA treatment & -0.02 & $(-0.03,-0.01)$ & $<0.001^{*}$ \\
\hline \multicolumn{4}{|l|}{ rtPA dose } \\
\hline Standard dose & Reference & & \\
\hline Modified dose & 0.20 & $(0.05,0.34)$ & $0.007^{*}$ \\
\hline $\begin{array}{l}\text { Charlson Comorbidity Index } \\
\text { (as a continuous variable) }\end{array}$ & -0.06 & $(-0.14,0.01)$ & $0.084^{*}$ \\
\hline
\end{tabular}

NIHSS: National Institute of Health Stroke Scale; rtPA: recombinant tissue plasminogen activator

T A B L E 4. Multiple linear regression analysis results of factors potentially impacting outcome in patients suffering middle cerebral artery ischemic stroke after rtPA treatment

\begin{tabular}{|c|c|c|c|c|c|c|}
\hline \multirow[t]{2}{*}{ Outcome } & \multicolumn{3}{|c|}{ BI effectiveness } & \multicolumn{3}{|c|}{ PASS effectiveness } \\
\hline & Coefficient $(\beta)$ & $95 \% \mathrm{CI}$ & $P$-Value & Coefficient $(\beta)$ & $95 \% \mathrm{CI}$ & $P$-value \\
\hline Age & -0.01 & $(-0.01,0.00)$ & 0.045 & -0.01 & $(-0.01,0.00)$ & 0.031 \\
\hline Rehabilitation type, Early mobilization & 0.23 & $(0.08,0.39)$ & 0.004 & 0.17 & $(0.03,0.32)$ & 0.019 \\
\hline NIHSS score at 24 hours after rtPA treatment & -0.02 & $(-0.03,0.00)$ & 0.006 & -0.01 & $(-0.02,0.00)$ & 0.043 \\
\hline
\end{tabular}

NIHSS: National Institute of Health Stroke Scale;

rtPA: recombinant tissue plasminogen activator

BI: Barthel Index

PASS: Postural Assessment Scale for Stroke

commencement of EM as well as age and NIHSS score at 24 hours post-rtPA may be associated with better functional improvement and postural control.

A previous study using a nationwide database showed that very early rehabilitation was associated with a significant increase functional independence in patients after treatment with rtPA [19]. However, in that study, the very early rehabilitation group included those who received any type or intensity of rehabilitation, including physical and occupational therapy and no detailed rehabilitation programs. In addition, patients treated with rtPA included in AVERT demonstrated a modest change in NIHSS score 24 hours post-rtPA [30]. Conversely, we could identify the exact timing of admission, the rtPA dosage and the starting date of out-of-bed activities by the EMR in our study. Moreover, the definition of "early" differed from that in our study: our mean time to a first mobilization in the EM group was 2.7 days, which was longer than the 22.4 hours for the usual care group in AVERT [21].

The results showed that starting out-of-bed mobilization within 24-72 hours of onset after rtPA treatment rather than later after ICU discharge resulting in facilitating functional recovery; however, our findings still need to be confirmed by future studies using a randomized controlled trial. These results are in agreement with findings of other studies that suggest that out-of-bed activities should commence early after stroke after receiving the rtPA treatment for better functional 
outcomes [19, 31]. Functional or motor recovery from a stroke event occurs through a combination of spontaneous and experience-dependent processes [32]. The effects of early mobilization may be rooted in the extreme sensitivity of the sensorimotor experiences due to the use of a limited time window during the acute stage of stroke [33, 34]. Enhancing motor experiences or providing greater vertical stimulation through active out-of-bed sitting and standing constituted early mobilization is thought to play an important role in functional recovery after stroke [34].

In addition, age and the NIHSS score at 24 hours after rtPA treatment were predictive of functional improvement in our study. The functional outcomes after acute stroke survivors are mainly influenced by the patients' age due to the lower functional reserve and the more advanced comorbidities [35, 36]. Moreover, stroke severity based on the NIHSS score at admission is as an independent predictor of post-stroke outcome, but patients showed a significant early improvement of 0.5-4 or more points in terms of NIHSS scores after rtPA treatment [37]. Early neurological recovery has been identified as a significant predictor of long-term outcome in thrombolysis studies [38, 39]. Therefore, in our study, it was also found that the NIHSS score at 24 hours post-rtPA, compared to the NIHSS score upon admission, is predictive of better functional improvement. A prompt neurological improvement has shown to be more accurate as the baseline NIHSS score increases [38]. The result indicate that future related prospective studies might need to consider including the 24-hour post-rtPA NIHSS score as a predictive factor for the functional outcome improvement after the stroke.

\section{Limitations}

First, this study was a retrospective correlation study, therefore we could not make a definitive causal claim about the impact of early mobilization. Second, the subjects were recruited from a single center, and there were no long-term follow-up examinations. However, a homogeneous group of patients was analyzed. Third, the actual length of each rehabilitation time was not obtained in this study. Finally, the small sample size of the study and the associated medical records confined the determination of all possible potential confounding effects [40, 41]. Besides, the study population was heterogeneous in their nutrition status and cognitive status after stroke, which may have influenced the functional outcomes.

\section{Conclusions}

Early mobilization commencement within 24-72 hours after MCA-IS treated with rtPA might predispose to better functional outcomes during the acute phase. Future studies will need to examine the early mobilization intervention for such an approach and to estimate its effectiveness within IS treated with rtPA.

\section{AUTHOR CONTRIBUTIONS}

Yen $\mathrm{H}-\mathrm{C}$ takes responsibility for the content of the manuscript, including the data and analysis. Yen H-C and Chuang W-
$\mathrm{Y}$ had full access to all of the data in the study and takes responsibility for the integrity of the data and the accuracy of the data analysis, including and especially any adverse effects. Yen H-C, Jeng J-S and Chen W-S contributed substantially to the study design, data analysis and interpretation, and the writing of the manuscript. All authors approved the final manuscript.

\section{ETHICS APPROVAL AND CONSENT TO PARTICIPATE}

The study was approved by the Institutional Review Board (IRB) of NTUH (ID number: 201801125RINA).

\section{ACKNOWLEDGMENT}

The authors thank staff at the NTUH stroke center (4D1) for supporting this study. The authors express gratitude to the Statistical Center of NTUH.

\section{FUNDING}

This study was supported by grants from the National Taiwan University Hospital (NTUH.108-s-4122).

\section{CONFLICT OF INTEREST}

The authors declare no potential conflicts of interest with respect to the research, authorship, and/or publication of this article.

\section{REFERENCES}

[1] Yasaka M, O'Keefe GJ, Chambers BR, Davis SM, Infeld B, O'Malley $\mathrm{H}$, et al. Streptokinase in acute stroke: effect on reperfusion and recanalization. Australian Streptokinase Trial Study Group. Neurology. 1998; 50: 626-632.

[2] Jauch EC, Saver JL, Adams HP, Bruno A, Connors JJB, Demaerschalk $\mathrm{BM}$, et al. Guidelines for the early management of patients with acute ischemic stroke: a guideline for healthcare professionals from the American Heart Association/American Stroke Association. Stroke. 2013; 44: 870-947.

[3] Ha J, Churilov L, Linden T, Bernhardt J. Bed rest or mobilization after rt-PA? A case-crossover study of factors influencing clinical decision making in stroke services. International Journal of Stroke. 2013; 8: 172179.

[4] Diserens K, Moreira T, Hirt L, Faouzi M, Grujic J, Bieler G, et al. Early mobilization out of bed after ischaemic stroke reduces severe complications but not cerebral blood flow: a randomized controlled pilot trial. Clinical Rehabilitation. 2012; 26: 451-459.

[5] Cumming TB, Thrift AG, Collier JM, Churilov L, Dewey HM, Donnan GA, et al. Very early mobilization after stroke fast-tracks return to walking: further results from the phase II AVERT randomized controlled trial. Stroke. 2011; 42: 153-158.

[6] Bernhardt J, Dewey H, Collier J, Thrift A, Lindley R, Moodie M, et al. A very early rehabilitation trial (AVERT). International Journal of Stroke. 2008; 1: 169-171.

[7] Horn SD, DeJong G, Smout RJ, Gassaway J, James R, Conroy B. Stroke rehabilitation patients, practice, and outcomes: is earlier and more aggressive therapy better? Archives of Physical Medicine and Rehabilitation. 2005; 86: S101-S114.

[8] Langhorne P, Stott D, Knight A, Bernhardt J, Barer D, Watkins C. Very 
early rehabilitation or intensive telemetry after stroke: a pilot randomised trial. Cerebrovascular Diseases. 2010; 29: 352-360.

[9] Indredavik B, Bakke F, Slordahl SA, Rokseth R, Hâheim LL. Treatment in a combined acute and rehabilitation stroke unit: which aspects are most important? Stroke. 1999; 30: 917-923.

[10] Zhang P, Zhang Q, Pu H, Wu Y, Bai Y, Vosler PS, et al. Very early-initiated physical rehabilitation protects against ischemic brain injury. Frontiers in Bioscience. 2012; 4: 2476-2489.

[11] Zhang A, Bai Y, Hu Y, Zhang F, Wu Y, Wang Y, et al. The effects of exercise intensity on $\mathrm{p}-\mathrm{NR} 2 \mathrm{~B}$ expression in cerebral ischemic rats. The Canadian Journal of Neurological Sciences. 2012; 39: 613-618.

[12] Zhang Y, Zhang P, Shen X, Tian S, Wu Y, Zhu Y, et al. Early exercise protects the blood-brain barrier from ischemic brain injury via the regulation of MMP-9 and occludin in rats. International Journal of Molecular Sciences. 2013; 14: 11096-11112.

[13] Lee M, Kim H, Kim S, Lee T, Lim B, Chang H, et al. Treadmill exercise suppresses ischemia-induced increment in apoptosis and cell proliferation in hippocampal dentate gyrus of gerbils. Life Sciences. 2003; 73: 2455 2465.

[14] Zhang L, Hu X, Luo J, Li L, Chen X, Huang R, et al. Physical exercise improves functional recovery through mitigation of autophagy, attenuation of apoptosis and enhancement of neurogenesis after MCAO in rats. BMC Neuroscience. 2013; 14: 46.

[15] Kim M, Bang M, Han T, Ko Y, Yoon B, Kim J, et al. Exercise increased $\mathrm{BDNF}$ and trkB in the contralateral hemisphere of the ischemic rat brain. Brain Research. 2005; 1052: 16-21.

[16] Luo CX, Jiang J, Zhou QG, Zhu XJ, Wang W, Zhang ZJ, et al. Voluntary exercise-induced neurogenesis in the postischemic dentate gyrus is associated with spatial memory recovery from stroke. Journal of Neuroscience Research. 2007; 85: 1637-1646.

[17] Bernhardt J, Chitravas N, Meslo IL, Thrift AG, Indredavik B. Not all stroke units are the same: a comparison of physical activity patterns in Melbourne, Australia, and Trondheim, Norway. Stroke. 2008; 39: 20592065.

[18] Kwakkel G, Wagenaar RC, Koelman TW, Lankhorst GJ, Koetsier JC. Effects of intensity of rehabilitation after stroke. A research synthesis. Stroke. 1997; 28: 1550-1556.

[19] Momosaki R, Yasunaga H, Kakuda W, Matsui H, Fushimi K, Abo M. Very early versus delayed rehabilitation for acute ischemic stroke patients with intravenous recombinant tissue plasminogen activator: a nationwide retrospective cohort study. Cerebrovascular Diseases. 2016; 42: 41-48.

[20] Muhl L, Kulin J, Dagonnier M, Churilov L, Dewey H, Lindén T, et al. Mobilization after thrombolysis (rtPA) within 24 hours of acute stroke: what factors influence inclusion of patients in a very Early Rehabilitation Trial (AVERT)? BMC Neurology. 2014; 14: 163.

[21] Ringleb PA, Bousser M, Ford G, Bath P, Brainin M, Caso V, et al. Guidelines for management of ischaemic stroke and transient ischaemic attack 2008. Cerebrovascular Diseases. 2008; 25: 457-507.

[22] Matsuda S, Ishikawa KB, Kuwabara K, Fujimori K, Fushimi K, Hashimoto H. Development and use of the Japanese case-mix system. Eurohealth. 2008; 14: 27-30.

[23] Winstein CJ, Stein J, Arena R, Bates B, Cherney LR, Cramer SC, et al. Guidelines for adult stroke rehabilitation and recovery: a guideline for healthcare professionals from the American Heart Association/American Stroke Association. Stroke. 2016; 47: e98-e169.

[24] Benaim C, Pérennou DA, Villy J, Rousseaux M, Pelissier JY. Validation of a Standardized Assessment of Postural Control in Stroke Patients. Stroke. 1999; 30: 1862-1868.

[25] Mahoney FI, Barthel DW. Functional evaluation: the Barthel index. Maryland State Medical Journal. 1965; 14: 61-65.

[26] Al-Khaled M, Matthis C, Eggers J. Predictors of in-hospital mortality and the risk of symptomatic intracerebral hemorrhage after thrombolytic therapy with recombinant tissue plasminogen activator in acute ischemic stroke. Journal of Stroke and Cerebrovascular Diseases. 2014; 23: 7-11.

[27] Wahlgren N, Ahmed N, Eriksson N, Aichner F, Bluhmki E, Dávalos A, et al. Multivariable analysis of outcome predictors and adjustment of main outcome results to baseline data profile in randomized controlled trials: Safe Implementation of Thrombolysis in Stroke-MOnitoring STudy (SITS-most). Stroke. 2008; 39: 3316-3322.

[28] Quan H, Sundararajan V, Halfon P, Fong A, Burnand B, Luthi J, et al. Coding algorithms for defining comorbidities in ICD-9-CM and ICD-10 administrative data. Medical Care. 2005; 43: 1130-1139.

[29] Koh GC, Chen CH, Petrella R, Thind A. Rehabilitation impact indices and their independent predictors: a systematic review. BMJ Open. 2013; 3: e003483.

[30] Bernhardt J, Churilov L, Ellery F, Collier J, Chamberlain J, Langhorne P, et al. Prespecified dose-response analysis for a very early rehabilitation trial (AVERT). Neurology. 2016; 86: 2138-2145.

[31] Arnold SM, Dinkins M, Mooney LH, Freeman WD, Rawal B, Heckman MG, et al. Very early mobilization in stroke patients treated with intravenous recombinant tissue plasminogen activator. Journal of Stroke and Cerebrovascular Diseases. 2015; 24: 1168-1173.

[32] Murphy TH, Corbett D. Plasticity during stroke recovery: from synapse to behaviour. Nature Reviews Neuroscience. 2009; 10: 861-872.

[33] Nudo RJ, Friel KM. Cortical plasticity after stroke: implications for rehabilitation. Revue Neurologique. 1999; 155: 713-717.

[34] Krakauer JW, Carmichael ST, Corbett D, Wittenberg GF. Getting neurorehabilitation right: what can be learned from animal models? Neurorehabilitation and Neural Repair. 2012; 26: 923-931.

[35] Riachy M, Sfeir F, Sleilaty G, Hage-Chahine S, Dabar G, Bazerbachi T, et al. Prediction of the survival and functional ability of severe stroke patients after ICU therapeutic intervention. BMC Neurology. 2008; 8: 24.

[36] Ducci RD, Lange MC, Zétola VDHF. Predictors of in-hospital mortality and dependence at discharge in patients with MCA stroke with intravenous thrombolysis. Internal and Emergency Medicine. 2017; 12: 453-460.

[37] Kablau M, Alonso A, Hennerici MG, Fatar M. Treatment with tPA predicts better outcome even if MCA occlusion persists. International Journal of Stroke. 2013; 8: 496-502.

[38] Yeo LL, Paliwal P, Teoh HL, Seet RC, Chan BP, Wakerley B, et al. Early and continuous neurologic improvements after intravenous thrombolysis are strong predictors of favorable long-term outcomes in acute ischemic stroke. Journal of Stroke and Cerebrovascular Diseases. 2013; 22: e590e596.

[39] Kharitonova T, Mikulik R, Roine RO, Soinne L, Ahmed N, Wahlgren N. Association of early National Institutes of Health Stroke Scale improvement with vessel recanalization and functional outcome after intravenous thrombolysis in ischemic stroke. Stroke. 2011; 42: 16381643.

[40] Spiotta AM, Vargas J, Hawk H, Turner R, Chaudry MI, Battenhouse H, et al. Impact of the ASPECT scores and distribution on outcome among patients undergoing thrombectomy for acute ischemic stroke. Journal of Neurointerventional Surgery. 2015; 7: 551-558.

[41] Campbell BCV, Donnan GA, Lees KR, Hacke W, Khatri P, Hill MD, et al. Endovascular stent thrombectomy: the new standard of care for large vessel ischaemic stroke. The Lancet Neurology. 2015; 14: 846-854.

How to cite this article: Hsiao-Ching Yen, Jiann-Shing Jeng, Wen-Ying Chuang, Wen-Shiang Chen. Is early mobilization associated with functional improvement in acute middle cerebral artery ischemic stroke treated with recombinant tissue plasminogen activator? A proof of concept and retrospective single-center study. Signa Vitae. 2021;17(2):174-180. doi:10.22514/sv.2021.016. 